\title{
Study of the Crystalline and Mesoporous Structure of a Heterogeneous Catalyst (ZSM-5) in Relation to Al, Si and Other Heteroatom, Distribution by Transmission Electron Microscopy
}

\author{
Y.C. Wang ${ }^{1}$, M. Kulzick ${ }^{1}$ and Anna Carlsson ${ }^{2}$ \\ 1. BP Amoco Chemical Co, 150 W. Warrenville Rd., Naperville, IL 60563, USA \\ 2. Thermo Fisher Scientific, Achtseweg Noord 5, 5651 GG Eindhoven, The Netherlands
}

The applications of HRTEM to electron-sensitive zeolites have been severely limited due to electron irradiation damage. Since the zeolite crystal structure is very complex even at low-index zone axis, observing the fine structure of zeolite framework requires a much higher resolution (1). The maximum spatial frequency contributing to the HRTEM image of electron sensitive crystalline materials is limited by either the SNR (signal-to-noise ratio) of the acquired image or crystallinity maintained during a TEM investigation. Higher accelerating voltage reduces electron irradiation damage from radiolysis because of the lower inelastic scattering probability; however it deteriorates the sensitivity of CCD cameras and phase contrast (scrambled phase at HRTEM). Therefore, higher electron dose is required to obtain enough SNR with higher accelerating voltages. On the other hand, a lower accelerating voltage is advantageous for the efficiency of electron detection, but works disadvantageously in electron irradiation damage. Since the damage process for zeolites proceeds by knock-on processes and radiolysis in the range of all practical accelerating voltages, operating at lower accelerating voltages has been proved to increase the rate of damage (3).

Zeolite frameworks are built from TO4 tetrahedral (T: $\mathrm{Si}$, or $\mathrm{Al}$, or other heteroatoms) to produce nanochannels or nano-cavities inside the crystals. Information about the size and shape of nanochannels or nano-cavities is very important in various zeolite applications. HRTEM has been demonstrated as a powerful tool for nanostructure analysis in catalyst research since it can provide structure information at molecular and atomic levels. Our research is to study the crystalline and mesoporous fine structure of a heterogeneous catalyst (ZSM-5 based zeolite) in relation to Al, other heteroatoms, and Si distribution by comparing a traditional TEM (FEI Osiris TEM) operated at lower accelerating voltage (80kV) for gaining better electron detection efficiency of the CCD camera, a FEI Titan Cs image corrected ETEM operated at $300 \mathrm{keV}$, and a new direct phase imaging technique, called the Integrated Differential Phase Contrast (iDPC) STEM (2) operated at $300 \mathrm{keV}$. The commercially available zeolite (Alfa Aesar $\mathrm{NH}_{4} \mathrm{ZSM}-5$, SAR-23, calcined for one hour at $550^{\circ} \mathrm{C}$ to form HZSM-5) sample was prepared by crushing the crystalline in an agate mortar, and then collected on a holey-Carbon coated $\mathrm{Cu}$ grid.

Fig. 1 shows a TEM image of a ZSM-5 sample taken at $80 \mathrm{keV}$ and a dose rate of around 50 electron $/ \AA^{2} \cdot$ second on a traditional non-corrected TEM. A Gatan Orius camera was used to acquire the image. The open channels of ZSM-5 framework structure could be visualized as the [010] projection from a large irregular plate-like crystal. Fig. 2 shows a TEM image of the ZSM-5 sample acquired on a Titan ETEM with Cs image corrector at 300kV. The direct view Gatan K2 Summit camera was used to acquire the images at counting mode with a dose rate of less than 10 electron/ $\AA^{2} \cdot$ second. The crystals are also on the [010] projection. Fig. 3 shows an iDPC-STEM image taken on a Thermo Scientific's probe corrected Themis system at $300 \mathrm{kV}$ with a dose of 260 electron $/ \AA^{2}$. 
The iDPC-STEM direct phase imaging technique provides a way to reveal all nanochannels in the ZSM5 crystal structure and, in our opinion, offers a preferred method for imaging zeolites [4].

References:

[1] K. Yoshida, Y. Sasaki, and H. Kurata, AIP Adv. 3 (2013), p. 042113.

[2] I. Lazić et al, Microscopy and Microanalysis 22 (Supplement 3) (2016), p. 36.

[3] R. Egerton, Microscopy Research \& Technique, 75(11) (2012), p. 1550.

[4] The authors gratefully acknowledge the Center for Functional Nanomaterials (CFN) of Brookhaven National Laboratory for granting the access of Titan ETEM with Cs image corrector under the CFN user proposal.
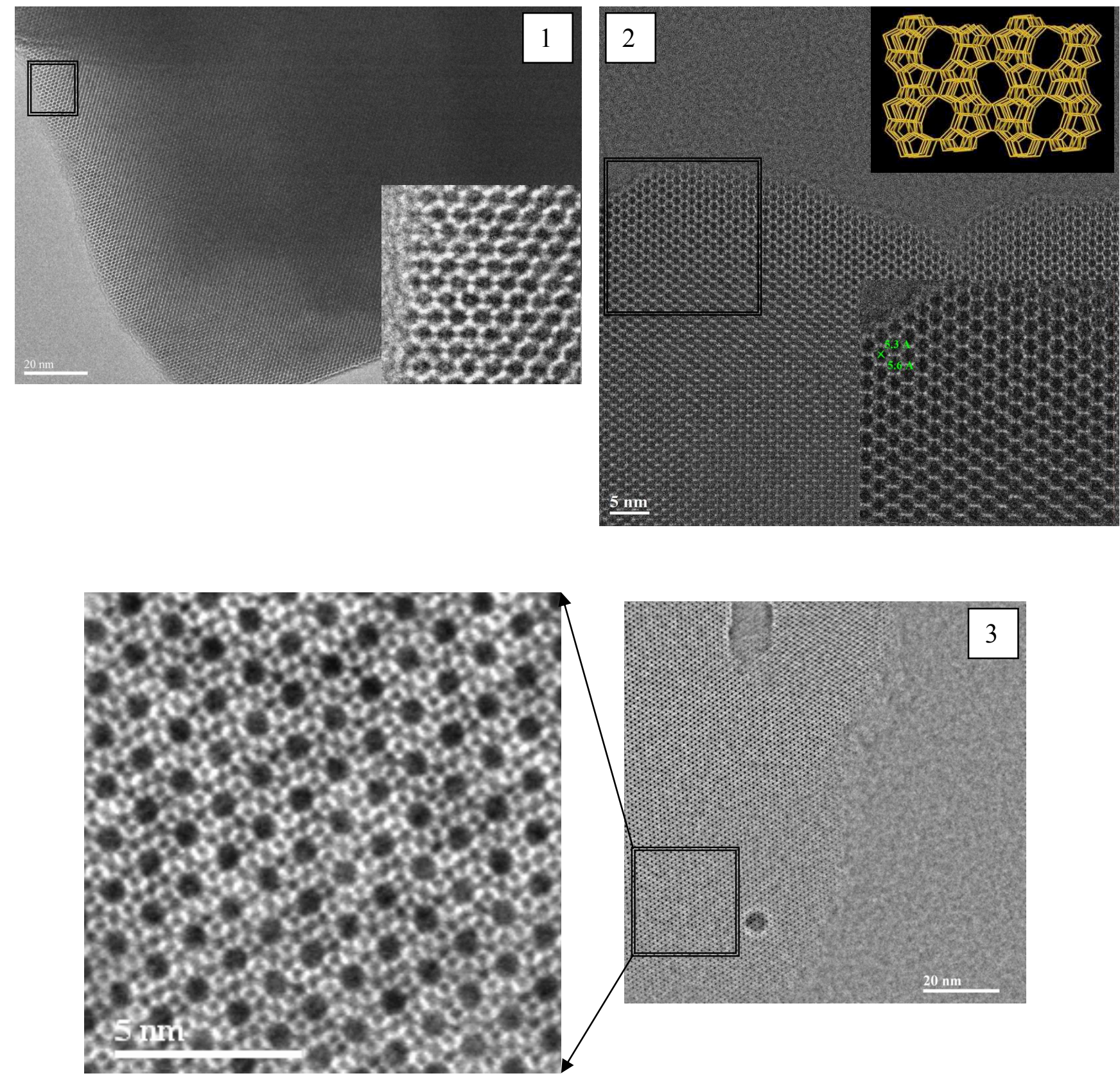